\title{
Controllable production of Janus ligaments by AC fields in a flow-focusing junction
}

\author{
Elena Castro-Hernández • Pablo García-Sánchez • Marta \\ León-Rodríguez • David Fernandez Rivas • Antonio Ramos
}

Received: date / Accepted: date

\begin{abstract}
We report the production of bicomponent Janus filaments of miscible aqueous fluids in a microfluidic electro-flow focusing device under the action of an AC electric field. The production of liquid filaments can lead to the generation of microfibers by adding a subsequent process of polymerization. Janus microfibers are of paramount importance in biomedical applications such as tissue production on crimped scaffolds. We show that the filament length is a function of the frequency signal, voltage amplitude and of the viscosity and conductivity of the dispersed phase. In particular, Janus filaments with diameters $\sim 10 \mu \mathrm{m}$ and longer than $1 \mathrm{~mm}$ are produced by $\mathrm{AC}$ voltages with frequencies below $150 \mathrm{kHz}$ and a viscosity of the dispersed phase $\sim 10 \mathrm{cP}$.
\end{abstract}

Keywords AC electric field · flow focusing - microfluidics · Janus

Elena Castro-Hernández · Marta León-Rodríguez

Área de Mecánica de Fluidos, Departamento de Ingeniería Aeroespacial y Mecánica de Fluidos, Universidad de Sevilla, Avenida de los Descubrimientos s/n 41092, Sevilla, Spain.

E-mail: elenacastro@us.es

Pablo García-Sánchez · Antonio Ramos

Departamento de Electrónica y Electromagnetismo, Facultad de Física, Universidad de Sevilla, Avenida de Reina Mercedes s/n, 41012 Sevilla, Spain.

E-mail: ramos@us.es

David Fernández Rivas

Mesoscale Chemical Systems Group, 7500 AE Enschede, The Netherlands.

\section{Introduction}

Bicomponent polymer fibers combine the properties of two different polymers to produce materials with new and improved properties that do not exist in either polymer fiber by itself. Considering a two-compartment system, the simplest structures are: (i) core-shell (with different interior and exterior) and (ii) Janus (also called side-by-side) structure, where the sides of the structure are different. Unlike core/shell architectures, the two functional materials in Janus structures are directly exposed to the surface so that the two functions can be displayed without reservation. Taking advantage of the special structure, many Janus design materials were studied, such as Janus particles (Perro et al., 2005; Wu et al., 2018), Janus disks (Liu et al., 2013), Janus sheets (Liu et al., 2015), Janus fibers (Gupta and Wilkes, 2003) and Janus ellipsoids (Kang et al., 2016).

Biocompatible fibers are capable of adapting to the physiological conditions of the human body and have become increasingly important for clinical applications. Fiber mats offer particular advantages due to their large surface area and their sorption/release properties. If loaded with drugs, delivery properties can be tailored to a specific release rate. Side-by-side bicomponent fibers have additional advantages, such as differential drug release properties, added surface due to different dissolution speeds under biological conditions, different morphologies, etc (Buschle-Diller et al., 2007).

It is well known that highly desirable crimp contraction properties can be achieved by Janus fibers since difference in shrinkage between the two polymers causes the self-crimping of bicomponent fibers. Tissue production on crimped fibrous scaffolds improves when compared with scaffolds containing uncrimped (straight) fibres 
(Surrao et al., 2012). Crimped fiber scaffolds may also be useful for the generation of cultured ligament tissue since ligaments with different crimp patterns present reduced mechanical strength (Chen et al., 2014).

The most widely used technique for the production of Janus fibers is the simultaneous electrospinning of two polymer solutions in a side-by-side fashion (Gupta and Wilkes, 2003; Chen et al., 2015; Starr and Andrew, 2013; Starr et al., 2015). Electrospinning is a process to easily produce polymeric fibers in the average diameter range of $100 \mathrm{~nm}$ to $5 \mathrm{~mm}$. The average diameter of the fibers produced in this way is at least one or two orders of magnitude smaller than the conventional fiber production methods like melt or solution spinning. As a result, these fibers possess a high aspect ratio that leads to a larger specific surface. However, concerns have been raised in relation to the ratio of the dominant component to the trace component varied along the length of the fibers (Gupta and Wilkes, 2003). Additionally, the produced fiber is of low orientation and crystallinity and thus, has little tenacity (Buschle-Diller et al., 2007).

Droplet and jet generation methods can be classified as passive or active, depending on the use of external actuation (Zhu and Wang, 2017). Techniques such as cross-flow, co-flow, flow-focusing (Gañán-Calvo, 1998; Pérez-Rigueiro et al., 2018), and step emulsification are grouped as passive. These techniques have been previously used to produce Janus droplets (Nisisako, 2016). The active techniques encompass the use of electric, magnetic, or centrifugal fields, just to name a few. In this work, we generate bicomponent Janus microjets of miscible fluids by combining a passive technique to produce an incipient jet and an active one to modulate the jet length. The former is achieved with a planar microfluidic flow-focusing junction (Anna et al., 2003), whereas the latter is accomplished by the application of an AC electric field. The advantage of using an electric field is almost instantaneous control of the features of droplet and jet production without altering the flow parameters (flow rate, viscosity, surface tension, etc).

AC electro-flow focusing devices have been previously used to control the production of droplets (Tan et al., 2014; Castro-Hernández et al., 2017) and long jets (CastroHernández et al., 2015, 2016). Tan et al. (2014) studied in depth the influence of the voltage amplitude, signal frequency and conductivity of the dispersed phase on the droplet size and production frequency. They found out that the droplet size can easily be modulated by tuning the voltage amplitude. We propose here the use of these devices to control the Janus jet length and, consequently, after a polymerization process Janus microfibers of desired length and/or Janus microparticles of desired size can be obtained.

\section{Experimental setup}

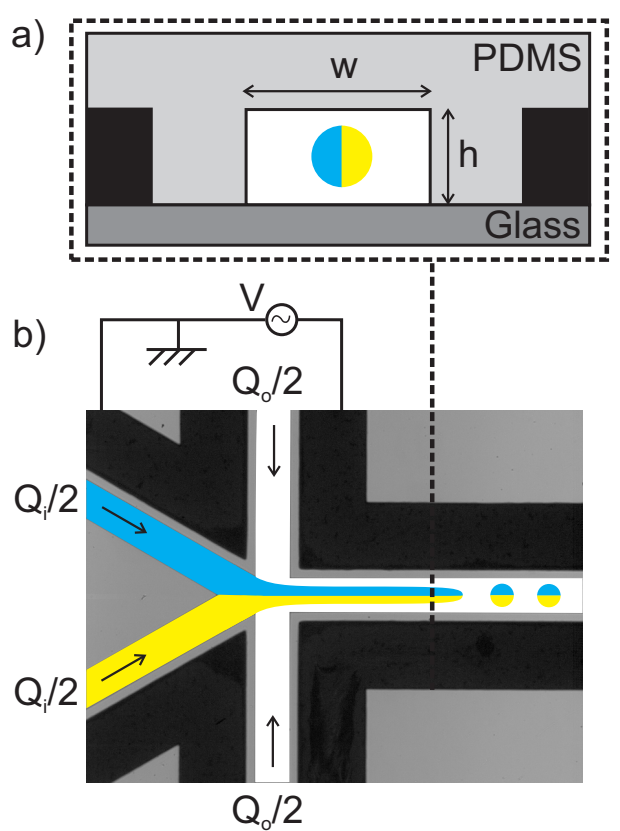

Fig. 1 a) Cross-section of the device at the level of the outlet microchannel $(w=100 \mu \mathrm{m}, h=35 \mu \mathrm{m})$. b) Microfluidic electro-flow focusing device under an AC electric field. The electrodes are in black, the upper inner dispersed phase in blue, the lower inner dispersed phase in yellow and the continuous phase in white.

We have redesigned our previous devices (Tan et al., 2014; Castro-Hernández et al., 2015, 2016, 2017) by having two inner inlets instead of one and allow for the production of bicomponent Janus microjets of miscible fluids. We have used soft lithography techniques to fabricate a microfluidic electro-flow focusing device by replica molding in polydimethylsiloxane (PDMS, Dow Corning, relative permittivity $\left.\varepsilon_{\mathrm{r}, \mathrm{PDMS}}=2.5\right)$. Figure 1 shows a schematic view of the device with channels of rectangular cross-section $w=100 \mu \mathrm{m}$ wide and $h=$ $35 \mu \mathrm{m}$ tall. The two channels for the two dispersed phases form a $30^{\circ}$ angle with the direction of the outlet channel whereas the two channels for the only continuous phase intersect perpendicularly at the junction. Two sets of electrodes are patterned on both sides of the junction and they are produced using the microsolidics technique (Siegel et al., 2006). The PDMS device is plasma bonded to a glass slide. A water-in-oil (W/O) 
emulsion is produced by focusing two inner aqueous stream (dispersed phases) with two outer oil streams (continuous phase). The inner and outer flow rates, $Q_{\mathrm{i}}$ and $Q_{\mathrm{o}}$ respectively, are controlled by means of two double syringe pumps (Model 33, Harvard Apparatus) and for most of our experiments they are fixed to $Q_{\mathrm{i}}=$ $50 \mu \mathrm{l} / \mathrm{h}$ and $Q_{\mathrm{o}}=400 \mu \mathrm{l} / \mathrm{h}$. The upper dispersed phase (blue) are black ink water and glycerol suspensions (Windsor \& Newton) with $\mathrm{KCl}$ whereas the lower dispersed phase (yellow) are $\mathrm{KCl}$ in Milli-Q water and glycerol. The upper and lower dispersed phases have identical viscosities $\left(\eta_{\mathrm{i}}=1 \mathrm{cP}, \eta_{\mathrm{i}}=6 \mathrm{cP}\right.$ or $\left.\eta_{\mathrm{i}}=13 \mathrm{cP}\right)$ and the same electrical conductivities varying between $\kappa=0.3 \mathrm{mS} / \mathrm{m}$ and $\kappa=30 \mathrm{mS} / \mathrm{m}$. The continuous phase (white) is mineral oil (RTM14, Sigma Aldrich) with a viscosity of $\eta_{\mathrm{o}}=100 \mathrm{cP}$. The relative permittivity of mineral oil is $\varepsilon_{\mathrm{r}, \mathrm{o}}=2.1$ and its electrical conductivity is negligible $\left(\kappa_{\mathrm{O}}<10^{-10} \mathrm{~S} / \mathrm{m}\right)$, being considered from now on as a perfect insulator. A $5 \%(\mathrm{w} / \mathrm{w})$ of a nonionic surfactant (Span 80, Sigma Aldrich) is added to the continuous phase lowering the surface tension of the liquid to liquid interface from $\sigma=40 \mathrm{mN} / \mathrm{m}$ to $\sigma=5 \mathrm{mN} / \mathrm{m}$, being this value independent of the ink, $\mathrm{KCl}$ and glycerol concentrations (Tan et al., 2014; Sinzato et al., 2017).

The high-voltage is applied to the downstream pair of electrodes while the others are grounded, which guarantees that the incoming liquid has zero potential (CastroHernández et al., 2015, 2016). As a consequence, there is an applied AC potential difference between this incoming liquid and the downstream electrodes. A sinusoidal voltage with frequencies varying from $f=1 \mathrm{kHz}$ to $f=200 \mathrm{kHz}$ (TGA1244, TTi) is amplified (PZD700A, Trek) resulting in voltage amplitudes from $V_{\mathrm{pp}}=400 \mathrm{~V}$ to $V_{\mathrm{pp}}=1000 \mathrm{~V}$. The microfluidic device is placed on an inverted microscope (Eclipse Ti-U, Nikon) connected to a high-speed camera (Phantom v7.3) with a resolution of $800 \times 256 \mathrm{px}^{2}$ when operated at an acquisition rate of $10^{4} \mathrm{fps}$. The jet length, $l_{\mathrm{j}}$, of at least 100 images is measured via image processing (Matlab, Mathworks).

\section{Results and discussion}

Figure 2 displays the dependence of jet length with signal frequency for given conductivity $(\kappa=25 \mathrm{mS} / \mathrm{m})$ and signal amplitude $\left(V_{\mathrm{pp}}=700 \mathrm{~V}\right)$. For comparison, we show in Figure 2(a) the situation where no voltage is applied.

Notice that no mixing between both inner dispersed phases occurs, as required for the production of bicomponet Janus filaments. The residence time of a fluid element can be defined as $t_{\mathrm{r}}=l_{\mathrm{j}} / U_{\mathrm{i}}$, where $l_{\mathrm{j}}$ is the

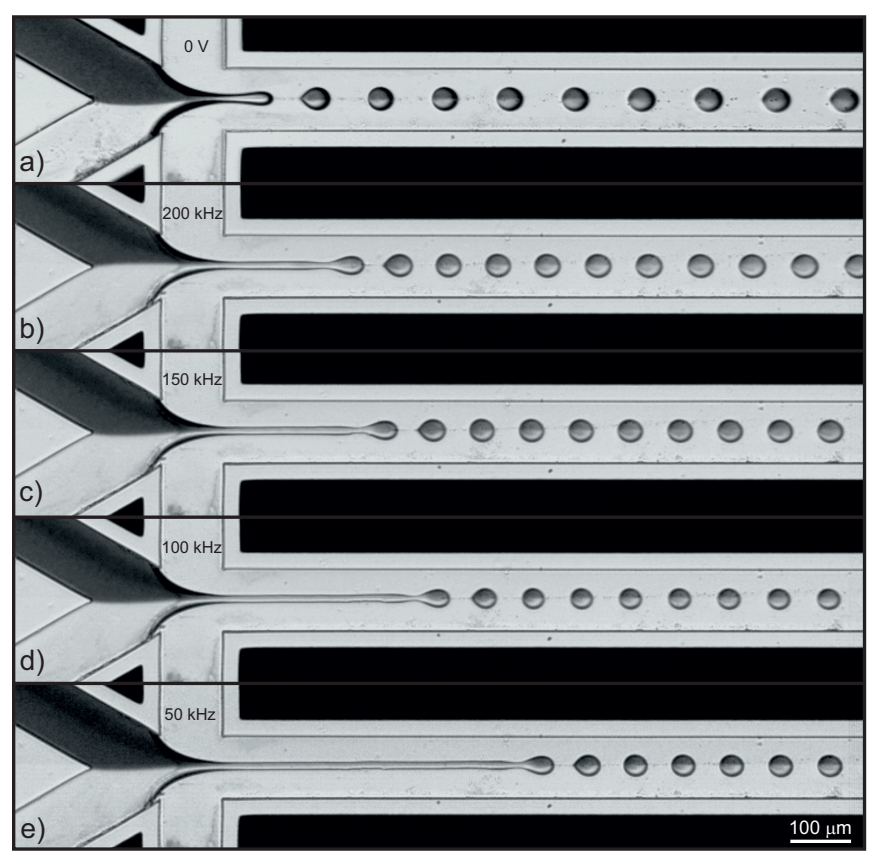

Fig. 2 Series of images showing the effect of decreasing the signal frequency on the jet length for $Q_{\mathrm{i}}=50 \mu \mathrm{l} / \mathrm{h}, Q_{\mathrm{o}}=$ $400 \mu \mathrm{l} / \mathrm{h}, \eta_{\mathrm{i}}=1 \mathrm{cP}, \eta_{\mathrm{o}}=100 \mathrm{cP}, \kappa=25 \mathrm{mS} / \mathrm{m}$ and $V_{\mathrm{pp}}=$ $700 \mathrm{~V}$ : a) $V_{\mathrm{pp}}=0 \mathrm{~V}$; b) $f=200 \mathrm{kHz}$; c) $f=150 \mathrm{kHz}$; d) $f=$ $100 \mathrm{kHz}$; e) $f=50 \mathrm{kHz}$.

jet length and $U_{\mathrm{i}}$ is the mean velocity of the dispersed phase. The diffusion time of a species can be estimated as $t_{\mathrm{d}}=d_{\mathrm{j}}^{2} / 4 D$, where $d_{\mathrm{j}}$ is the jet diameter and $D$ is the species diffusion coefficient. Ideally, to produce Janus ligaments the residence time should be much smaller than the diffusion time. This criterion allows us to define a maximum Janus jet length $l_{\mathrm{j}}=U_{\mathrm{i}} d_{\mathrm{j}}^{2} / 4 D$. The ink diffusion coefficient in water is $D \simeq 10^{-4} \mathrm{~mm}^{2} / \mathrm{s}$ at room temperature. In our experiments $U_{\mathrm{i}}=100 \mathrm{~mm} / \mathrm{s}$ and $d_{\mathrm{j}}=13 \mu \mathrm{m}$ leading to a maximum Janus jet length of $42 \mathrm{~mm}$, which is much longer than the jets we observed. Therefore, we do not expect any mixture between both dispersed phases as confirmed by our experimental observations.

In agreement to previous results (Castro-Hernández et al., 2015, 2016), longer jets are found as the signal amplitude is increased, the conductivity is incremented or the frequency of the signal is reduced. In addition, they experimentally observed a low frequency limit below which an unstable regime appears. This limit depends on the conductivity of the dispersed phase and on the applied voltage amplitude. Interestingly, Kim et al. (2007) observed long jets with DC fields, although this limit could not be reached by decreasing the frequency of an AC field.

Figure 3 depicts the jet length versus the signal fre- 
quency for different voltage amplitudes and $\kappa=10 \mathrm{mS} / \mathrm{m}$. The jet length is measured from the beginning of the downstream electrodes.

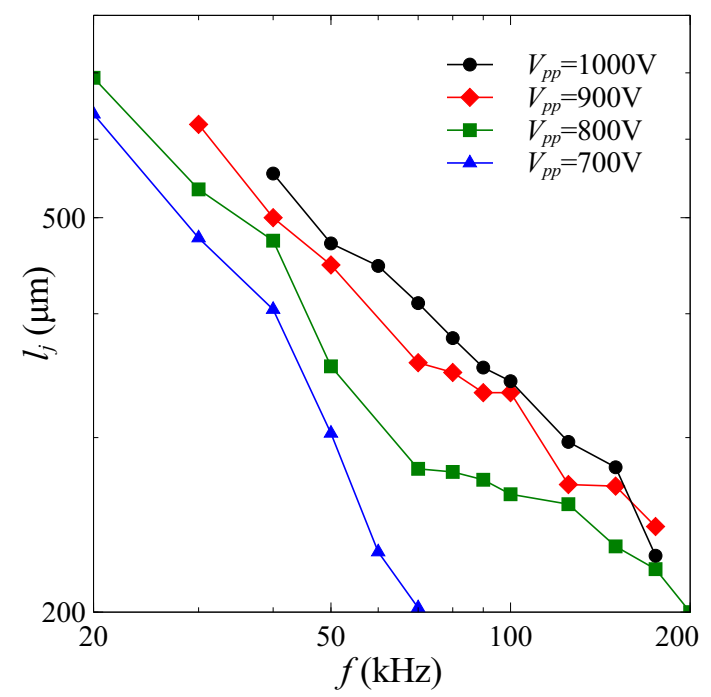

Fig. 3 Jet length versus the signal frequency for $Q_{\mathrm{i}}=$ $50 \mu \mathrm{l} / \mathrm{h}, Q_{\mathrm{o}}=400 \mu \mathrm{l} / \mathrm{h}, \eta_{\mathrm{i}}=1 \mathrm{cP}, \eta_{\mathrm{o}}=100 \mathrm{cP}, \kappa=10 \mathrm{mS} / \mathrm{m}$ and different voltage amplitudes.
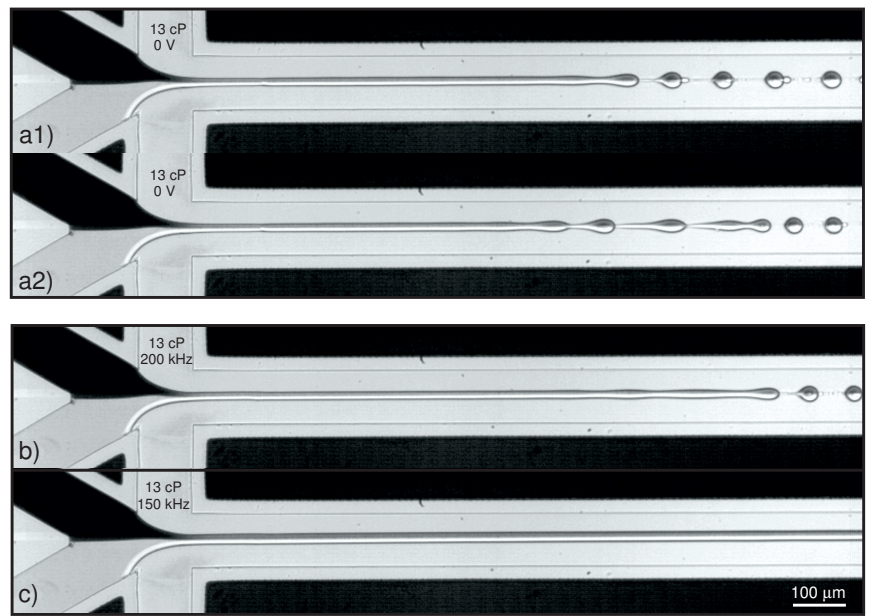

Fig. 4 Series of images showing the effect of decreasing the signal frequency on the jet length for $Q_{\mathrm{i}}=50 \mu \mathrm{l} / \mathrm{h}, Q_{\mathrm{o}}=$ $400 \mu \mathrm{l} / \mathrm{h}, \eta_{\mathrm{i}}=13 \mathrm{cP}, \eta_{\mathrm{o}}=100 \mathrm{cP}, \kappa=10 \mathrm{mS} / \mathrm{m}$ and $V_{\mathrm{pp}}=$ $400 \mathrm{~V}: \mathrm{a} 1) V_{\mathrm{pp}}=0 \mathrm{~V}$; a 2$) V_{\mathrm{pp}}=0 \mathrm{~V}$; b) $f=200 \mathrm{kHz}$; c) $f=$ $150 \mathrm{kHz}$.

Since many aqueous polymer solutions have viscosities greater than $1 \mathrm{cP}$, we have also experimentally investigated the effect of increasing the viscosity of the inner phase by adding glycerol. When the inner viscosity is raised from $\eta_{\mathrm{i}}=1 \mathrm{cP}$ to $\eta_{\mathrm{i}}=13 \mathrm{cP}$ longer jets are produced. Figures $4(\mathrm{a} 1)$ and $4(\mathrm{a} 2)$ are two frames of the jet breakup in the absence of electric field. According to Guillot et al. $(2007,2008)$ about confined jets at low Reynolds numbers, increasing the inner viscosity favours the jetting against the dripping regime, in agreement with our experimental observations. The irregular pinch-off position resembles a jet breakup due to convective instabilities. As expected, even longer jets are obtained upon application of an AC voltage. Figures $4(\mathrm{~b})$ and $4(\mathrm{c})$ illustrate the effect of decreasing the signal frequency on the jet length for given conductivity $(\kappa=10 \mathrm{mS} / \mathrm{m})$ and signal amplitude $\left(V_{\mathrm{pp}}=400 \mathrm{~V}\right)$. A jet longer than the camera field of view $\left(l_{\mathrm{j}}>1280 \mu \mathrm{m}\right)$ results for $f \leq 150 \mathrm{kHz}$.

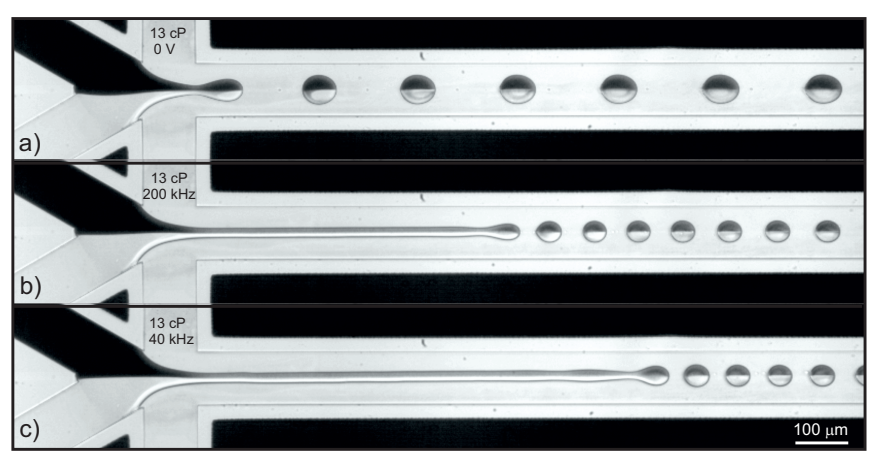

Fig. 5 Series of images showing the effect of decreasing the signal frequency on the jet length for $Q_{\mathrm{i}}=12.5 \mu \mathrm{l} / \mathrm{h}, Q_{\mathrm{o}}=$ $100 \mu \mathrm{l} / \mathrm{h}, \eta_{\mathrm{i}}=13 \mathrm{cP}, \eta_{\mathrm{o}}=100 \mathrm{cP}, \kappa=10 \mathrm{mS} / \mathrm{m}$ and $V_{\mathrm{pp}}=$ $500 \mathrm{~V}: \mathrm{a}) V_{\mathrm{pp}}=0 \mathrm{~V}$; b) $f=200 \mathrm{kHz}$; ) $f=40 \mathrm{kHz}$.

The jet undergoes great changes in length for the experiments with gycerol, in contrast with the observations for $\eta_{\mathrm{i}}=1 \mathrm{cP}$, which shows a more regular breakup. The difference between breakups may be explained in terms of linear stability analysis. Perturbations in absolute instabilities grow with time and space, downstream and upstream, and are independent of the external noise. Perturbations in convective instabilities are convected downstream and they depend on the external noise. Although it is a popular belief that jet breakup is always due to convective instabilities, Gañán-Calvo et al. (2006) and Utada et al. (2008) reported situations where long jets can experience breakups due to absolute instabilities. In those situations the jet breakup resembles a dripping regime but at the end of a long jet. This is similar to our observation for jet breakups with $\eta_{\mathrm{i}}=1 \mathrm{cP}$ in $\mathrm{AC}$ electric fields. In our experiments, the electric field amplitude on the surface of the jet decreases exponentially from the inlet. It has a stabilizing effect that dissapears as we go downstream below a certain field amplitude Castro-Hernández et al. (2015, 
2016). It seems that the jet breakup for experiments with $\eta_{\mathrm{i}}=1 \mathrm{cP}$ may occur due to absolute instability, while the breakup for jets with $\eta_{\mathrm{i}}=13 \mathrm{cP}$ may be due to convective instabilities. When the viscosity of the dispersed phase is increased a transition from the absolute to the convective region takes place, which might explain the changes observed in the jet length.

The experimental conditions above produce glycerol jets which are very long even in the absence of electric fields. Thus, under these conditions, application of $\mathrm{AC}$ voltages does not allow for a fine control of the jet length within a wide range. We have performed further experiments with the goal of recovering an incipient jet for zero voltage. To this end, we have followed the observations by Castro-Hernández et al. (2012) for a coflow configuration, where droplets of constant diameter emanate from a jet whose length is reduced by decreasing the inner and outer flow rates but keeping a constant ratio between them. In our current flow focusing geometry, we have reduced the flow rates to $Q_{\mathrm{i}}=12.5 \mu \mathrm{l} / \mathrm{h}$ and $Q_{\mathrm{o}}=100 \mu \mathrm{l} / \mathrm{h}$, obtaining jets of the same diameter but shorter. For these experimental conditions, Figure 5 displays the effect of decreasing the signal frequency on the jet length for given conductivity and voltage amplitude. For comparison, we show in Figure 5(a), the situation where no voltage is applied. This confirms that, reducing both inner and outer flow rates at constant $Q_{\mathrm{i}} / Q_{\mathrm{o}}=0.125$, we recover the control of the jet length from the inlet with the applied AC signal.

\section{Conclusions}

In summary, we report the production of Janus microjets of miscible fluids in a microfluidic electro-flow focusing device under the action of an AC electric field. The production of liquid filaments can lead to the generation of microfibers by adding a subsequent process of polymerization. Janus microfibers are of paramount importance in biomedical applications such as tissue production on crimped scaffolds. The length of the bicomponent filament can be finely controlled by tuning the frequency of the $\mathrm{AC}$ voltage. We studied the effect of increasing the inner fluid viscosity and, interestingly, we observed the appearance of a new regime that resembles an absolute-to-convective transition, in agreement with previous studies for a coflow configuration.

Acknowledgements The authors would like to acknowledge financial support from Spanish Government Ministry MEC under Contracts DPI2016-78887-C3-1-R and FIS2014-54539P. They would also like to acknowledge the technical assistance of S. Schlautman in the fabrication of the microfluidic devices.

\section{References}

Anna SL, Bontoux N, Stone HA (2003) Formation of dispersions using Flow Focusing in microchannels. Appl Phys Lett 82:364-366

Buschle-Diller G, Cooper J, Xie Z, Wu Y, Waldrup J, Ren X (2007) Release of antibiotics from electrospun bicomponent fibers. Cellulose 14(6):553-562

Castro-Hernández E, Campo-Cortés F, Gordillo JM (2012) Slender-body theory for the generation of micrometre-sized emulsions through tip streaming. Journal of Fluid Mechanics 698:423-445

Castro-Hernández E, García-Sánchez P, Tan SH, Gañán-Calvo AM, Baret JC, Ramos A (2015) Breakup length of ac electrified jets in a microfluidic flow-focusing junction. Microfluidics and Nanofluidics 19(4):787-794

Castro-Hernández E, García-Sánchez P, AlzagaGimeno J, Tan SH, Baret JC, Ramos A (2016) Ac electrified jets in a flow-focusing device: Jet length scaling. Biomicrofluidics 10(4):043,504

Castro-Hernández E, García-Sánchez P, VelencosoGómez A, Silas-Jurado A, Rivas DF, Ramos A (2017) Droplet group production in an ac electroflow-focusing microdevice. Microfluidics and nanofluidics 21(10):158

Chen F, Hayami JW, Amsden BG (2014) Electrospun poly (l-lactide-co-acryloyl carbonate) fiber scaffolds with a mechanically stable crimp structure for ligament tissue engineering. Biomacromolecules 15(5):1593-1601

Chen G, Xu Y, Yu DG, Zhang DF, Chatterton NP, White KN (2015) Structure-tunable janus fibers fabricated using spinnerets with varying port angles. Chemical Communications 51(22):4623-4626

Gañán-Calvo AM (1998) Generation of steady liquid microthreads and micron-sized monodisperse sprays in gas streams. Physical Review Letters 80(2):285

Gañán-Calvo AM, et al. (2006) Jetting-dripping transition of a liquid jet in a lower viscosity co-flowing immiscible liquid: the minimum flow rate in flow focusing. Journal of Fluid Mechanics 553:75-84

Guillot P, Colin A, Utada AS, Ajdari A (2007) Stability of a jet in confined pressure-driven biphasic flows at low Reynolds numbers. Phys Rev Lett 99:104,502

Guillot P, Colin A, Ajdari A (2008) Stability of a jet in confined pressure-driven biphasic flows at low Rey- 
nolds number in various geometries. Phys Rev E 78:016,307

Gupta P, Wilkes GL (2003) Some investigations on the fiber formation by utilizing a side-by-side bicomponent electrospinning approach. Polymer 44(20):63536359

Kang DW, Ko W, Lee B, Park BJ (2016) Effect of geometric and chemical anisotropy of janus ellipsoids on janus boundary mismatch at the fluid-fluid interface. Materials 9(8):664

Kim H, Luo D, Link D, Weitz DA, Marquez M, Cheng Z (2007) Controlled production of emulsion drops using an electric field in a flow-focusing microfluidic device. Appl Phys Lett 91(13):133,106

Liu J, Liu G, Zhang M, Sun P, Zhao H (2013) Synthesis and self-assembly of amphiphilic janus laponite disks. Macromolecules 46(15):5974-5984

Liu Y, Liang F, Wang Q, Qu X, Yang Z (2015) Flexible responsive janus nanosheets. Chemical Communications 51(17):3562-3565

Nisisako T (2016) Recent advances in microfluidic production of janus droplets and particles. Current Opinion in Colloid \& Interface Science 25:1-12

Pérez-Rigueiro J, Madurga R, Gañán-Calvo A, Plaza G, Elices M, López P, Daza R, González-Nieto D, Guinea G (2018) Straining flow spinning of artificial silk fibers: A review. Biomimetics 3(4):29

Perro A, Reculusa S, Ravaine S, Bourgeat-Lami E, Duguet E (2005) Design and synthesis of janus microand nanoparticles. Journal of materials chemistry 15(35-36):3745-3760

Siegel AC, Shevkoplyas SS, Weibel DB, Bruzewicz DA, Martinez AW, Whitesides GM (2006) Cofabrication of electromagnets and microfluidic systems in poly(dimethylsiloxane). Angew Chem 118:7031-7036

Sinzato YZ, Dias NJS, Cunha FR (2017) An experimental investigation of the interfacial tension between liquid-liquid mixtures in the presence of surfactants. Experimental Thermal and Fluid Science 85:370-378

Starr JD, Andrew JS (2013) Janus-type bi-phasic functional nanofibers. Chemical Communications 49(39):4151-4153

Starr JD, Budi MA, Andrew JS (2015) Processingproperty relationships in electrospun janus-type biphasic ceramic nanofibers. Journal of the American Ceramic Society 98(1):12-19

Surrao DC, Fan JC, Waldman SD, Amsden BG (2012) A crimp-like microarchitecture improves tissue production in fibrous ligament scaffolds in response to mechanical stimuli. Acta biomaterialia 8(10):37043713

Tan SH, Semin B, Baret JC (2014) Microfluidic flowfocusing in ac electric fields. Lab Chip 14(6):1099-
1106

Utada AS, Fernández-Nieves A, Gordillo JM, Weitz D (2008) Absolute instability of a liquid jet in a coflowing stream. Phys Rev Lett 100:014,502

Wu Q, Yang C, Yang J, Huang F, Liu G, Zhu Z, Si T, Xu RX (2018) Photopolymerization of complex emulsions with irregular shapes fabricated by multiplex coaxial flow focusing. Applied Physics Letters 112(7):071,601

Zhu P, Wang L (2017) Passive and active droplet generation with microfluidics: a review. Lab on a Chip Miniaturisation for Chemistry and Biology 17(1):3475 